hip circumference and leisure-time physical activity on all-cause mortality.

Methods We used a prospective population design with approximately 14 years' follow-up and estimated the HRs of all-cause mortality for combinations of physical activity and hip circumference. 3120 men and 4068 women aged 21 to 92 years without pre-existing diagnosis of diabetes, stroke, ischaemic heart disease, or cancer in 1991-1994 and with complete information on the variables of interest were included. They were followed until 2009 in the Danish Civil Registration System, with 1.5\% loss to follow-up and 2334 deaths.

Results Hip circumference was inversely associated with all-cause mortality irrespective of physical activity. However, physical activity seemed to counterbalance some of the adverse health effects of a small hip circumference, with the excess mortality in the lower quartile of hip circumference being reduced by $41 \%$ in men (HRdiff: $1.41,95 \%$ CI 1.14 to 1.74 ) and $40 \%$ in women (1.40, 1.14 to 1.71 ) when comparing physically inactive with the active. These associations were observed after adjustment for waist circumference, height, and weight change in the 6 months before the examination. Conclusion A small hip circumference appears hazardous to survival. However, being physically active may counterbalance some of the hazardous effects of a small hip circumference.

\section{P1-489 PREVALENCE AND DETERMINANTS OF SOLITARY PULMONARY NODULES DETECTED USING THORACIC IMAGING TESTS DURING ROUTINE CLINICAL PRACTICE}

doi:10.1136/jech.2011.142976g.77

\begin{abstract}
1,2B Lumbreras, ${ }^{3}$ I Gonzalez-Alvarez, ${ }^{4} \mathrm{~J}$ Vilar, ${ }^{1,2} \mathrm{~L}$ A Parker, ${ }^{* 1,2} \mathrm{M}$ A Pastor, ${ }^{1} \mathrm{~N}$ GomezSaez, ${ }^{3} \mathrm{~F}$ Lorente, ${ }^{4} \mathrm{M}$ L Domingo, ${ }^{3} \mathrm{~L}$ Perez, ${ }^{3} \mathrm{~N}$ Picazo. ${ }^{1}$ Department of Public Health, Alicante, Community of Valencia, Spain; ${ }^{2}$ CIBERESP (CIBER in Epidemiology and Public Health), Barcelona, Cataluña, Spain; ${ }^{3}$ Radiodiagnostic Service, San Juan de Alicante Hospital, Alicante, Community of Valencia, Spain; ${ }^{4}$ Radiodiagnostic Service, Dr Pesset Hospital, Valencia, Community of Valencia, Spain
\end{abstract}

Introduction The finding of a solitary pulmonary nodule (SPN) $(<3 \mathrm{~cm})$ in routine radiological examination may represent an early stage of lung cancer or a benign lesion, where any further diagnostic procedures may pose an unnecessary risk to patient health. Although SPNs have been described in high risk populations, their frequency in routine clinical care has not been determined.

Methods 4681 consecutive patients $\geq 35$ years referred for a thoracic imaging test in two hospitals in the Community of Valencia, Spain in 2010 were included. Six expert radiologists independently classified each imaging report according to the presence or absence of a SPN. Other variables, such as patient demographics diagnosis suspicion, smoking habit, the referral clinical department, type of radiological test performed, and clinical setting were ascertained from medical records. The association between SPNs and patient/ clinical characteristics was assessed with unconditional logistic regression.

Results SPNs were observed in 351 patients (7.5\%) and their prevalence varied according to patient characteristics and the referral department, with oncology $(15.4 \%)$, primary care $(10.7 \%)$ and pneumology $(9.8 \%)$, being the highest. After controlling for other factors, SPNs were more common in men (aOR 1.47, $95 \%$ CI 1.25 to 1.75 ), and in smokers (aOR $1.62,95 \%$ CI 1.41 to 1.82 ).

Conclusions At least one in every 13 patients undergoing a thoracic imaging test during routine clinical care will show a SPN. The follow-up of these patients is needed in order to estimate their predictive value for lung cancer, and avoid the initiation of an unnecessary cascade of clinical procedure in benign lesions.
P1-490 SOCIOECONOMIC INEQUALITIES IN HEIGHT, LEG LENGTH AND TRUNK LENGTH AMONG CHILDREN AGED 6.5 YEARS AND THEIR PARENTS FROM THE REPUBLIC OF BELARUS: EVIDENCE FROM THE PROMOTION OF BREASTFEEDING INTERVENTION TRIAL (PROBIT)

doi:10.1136/jech.2011.142976g.78

${ }^{1} \mathrm{R}$ Patel, ${ }^{* 1,2} \mathrm{D}$ A Lawlor, ${ }^{3} \mathrm{M}$ S Kramer, ${ }^{1,2} \mathrm{G}$ D Smith, ${ }^{4} \mathrm{~N}$ Bogdanovich, ${ }^{4} \mathrm{~L}$ Matush

${ }^{1,2} \mathrm{R}$ M Martin. 'School of Social and Community Medicine, University of Bristol, Bristol, UK; ${ }^{2}$ MRC Centre for Causal Analyses in Translational Epidemiology (CAiTE), University of Bristol, Bristol, UK; ${ }^{3}$ Department of Pediatrics and Department of Epidemiology, Biostatistics and Occupational Health, McGill University Faculty of Medicine, Montreal, Canada; ${ }^{4}$ The National Research and Applied Medicine Mother and Child Centre, Minsk, Belarus

Introduction Lower socioeconomic position is associated with shorter stature, in particular shorter leg length, but the magnitude of these associations in non-Western countries has received little attention.

Aim To examine socioeconomic differentials in height, leg and trunk length in 6.5 year olds from the Republic of Belarus and compare these to differentials in parental height.

Methods We used data from a cohort of 13889 children born in Belarus between June 1996 and December 1997 to investigate associations of parental educational attainment and highest household occupation with: a) measured child standing height, trunk and leg length at age 6.5 years; and b) the parents' reported standing height. Multivariable linear regression was used to examine associations.

Results Children from non-manual households were $1.1 \mathrm{~cm}(95 \% \mathrm{CI}$ 0.8 to $1.3 \mathrm{~cm}$ ) taller than those from manual households. Mothers and fathers from non-manual backgrounds were $0.7 \mathrm{~cm}(0.5$ to 0.8$)$ and $1.8 \mathrm{~cm}$ (1.6 to 2.0) taller than those from manual backgrounds, respectively. Associations with higher parental educational attainment were similar. The magnitudes of the associations of socioeconomic position with leg length were similar to those with trunk length. Adjusting for mid-parental height and number of older siblings attenuated children's associations markedly.

Conclusions In Belarus, similar socioeconomic differentials in height were observed in both children and their parents. Among children height differentials were partly explained by mid-parental height and number of older siblings. Leg length was not a more sensitive indicator of childhood socioeconomic conditions than trunk length.

\section{P1-491 SOCIO-ECONOMIC VARIATION IN THE USE OF CT SCANS IN} YOUNG PEOPLE IN THE NORTH OF ENGLAND, 1990-2002

doi:10.1136/jech.2011.142976g.79

${ }^{1} \mathrm{M}$ Pearce, ${ }^{* 1} \mathrm{~J}$ Salotti, ${ }^{2} \mathrm{~K}$ McHugh, ${ }^{3} \mathrm{~K}$ P Kim, ${ }^{1} \mathrm{~A}$ Craft, ${ }^{4} \mathrm{~J}$ Lubin, ${ }^{4} \mathrm{E}$ Ron, ${ }^{5} \mathrm{~L}$ Parker ${ }^{1}$ Newcastle University, Newcastle upon Tyne, UK; ${ }^{2}$ Great Ormond Street Hospital, London, UK; ${ }^{3}$ Kyung Hee University, Gyeongi-Do, Republic of Korea; ${ }^{4}$ National Cancer Institute, Bethesda, Maryland, USA; ${ }^{5}$ Dalhousie University, Halifax, Nova Scotia, Canada

Introduction Social patterning is known to influence health throughout life. In childhood, studies have shown increased injury rates in more deprived settings. Through this, it is also possible that socio-economic status may be related to rates of undergoing certain medical procedures with relatively high radiation doses, such as computed tomography (CT) scans. This study aimed to assess socioeconomic variation among young people having CT scans in the North of England between 1990 and 2002.

Methods Electronic data were obtained from Radiology Information Systems of all nine National Health Service hospital Trusts in the region. Data related to CT scans, including sex, date of scan, age at scan, number and type of scans were assessed in relation to quintiles of Townsend deprivation scores, obtained from linkage of postcodes with UK census data. 
Results During the study period, 39676 scans were recorded on 21089 patients. The number of scans and patients scanned differed in relation to quintiles of deprivation, with increasing numbers of scans and patients associated with increasing area-level deprivation. Significant associations were also seen between deprivation and age at scan, age at first scan, type of CT scan, and the number of scans per patient.

Conclusion Social inequalities exist in the numbers of young people undergoing CT scans with those from deprived areas more likely to do so. This is likely to reflect the rates of injuries in these individuals and implies that certain groups within the population may receive higher radiation doses than others due to medical procedures.

\section{P1-492 PREDICTORS OF CHILDHOOD PHYSICAL ACTIVITY: THE GATESHEAD MILLENNIUM STUDY}

doi:10.1136/jech.2011.142976g.80

${ }^{1} \mathrm{M}$ Pearce, ${ }^{* 1} \mathrm{~L}$ Basterfield, ${ }^{1} \mathrm{~K}$ Mann, ${ }^{1} \mathrm{~A}$ Adamson, ${ }^{1} \mathrm{~K}$ Parkinson, ${ }^{2} \mathrm{C}$ Wright, ${ }^{2} \mathrm{~J}$ Reilly. ${ }^{1}$ Newcastle University, Newcastle upon Tyne, UK; ${ }^{2}$ Glasgow University, Glasgow, UK

Introduction Conflicting evidence exists for associations between birth weight and childhood physical activity (PA) levels. It is important to know what other, potentially modifiable, factors influence PA in children given its' association with adiposity. Our aim was to identify predictors of childhood PA levels in the Gateshead Millennium Study (GMS), a population based cohort of 1029 infants born in 1999-2000 in Gateshead, Northern England.

Methods Throughout infancy and early childhood, detailed information was collected. Assessments at age 9 yrs included body composition, objective measures of habitual PA (using accelerometers during waking hours). Mean total volumes of PA (accelerometer count per minute, $\mathrm{cpm}$ ) and moderate-vigorous intensity PA (MVPA), and the percentage of time spent in sedentary behaviour (\% SB) were quantified and direct and mediating associations analysed within path models.

Results Significant differences were seen in all three outcomes between males and females $(p<0.001)$. No direct significant associations were seen with birth weight. Increased paternal age was associated with significant increases in \% SB and decreases in cpm and MVPA $(p<0.033)$. Associations with BMI at 9 yrs were in the expected directions. Increased time spent in sports clubs was significantly associated with decreased $\% \mathrm{SB}(\mathrm{p}=0.02)$ and increased MVPA $(p=0.01)$, but not cpm $(p=0.13)$.

Conclusions Although we found no evidence for an effect of birth weight on PA, path models suggest indirect effects mediated through BMI. Having an older father appeared to have a negative impact on the child's PA levels, while participation in sports clubs increases time spent in MVPA, but not cpm.

\section{P1-493 PROBABILISTIC RECORD LINKAGE: APPLICATION IN THE POPULATION-BASED CANCER REGISTRY OF SÃO PAULO (PBCR-SP), BRAZIL}

doi:10.1136/jech.2011.142976g.81

${ }^{1} S$ V Peres, ${ }^{* 1}$ M R D 0 Latorre, ${ }^{2}$ F A S Michels, ' $\mathrm{C}$ Terra. ${ }^{1}$ School of Public Health, University of São Paulo, São Paulo, Brazil; ${ }^{2}$ Population-Based Cancer Registry of São Paulo, School of Public Health, University of São Paulo, São Paulo, Brazil

Introduction Probabilistic record linkage is an alternative to different study designs because it generates, at lower operating cost, better database completeness. The aim of this study was to assess the performance of the probabilistic record linkage between PBCR-SP for cases of stomach and intestine cancer and São Paulo death database.
Methods From 1997 through 2005, 18511 cases were recorded by PBCR-SP. From the 1997 through 2007, 767752 deaths were recorded by Mortality Information System of São Paulo city. The fetal deaths were excluded. Exceeding spaces between characters were excluded. Type and size of field, and different spellings with same phoneme were standardised. Prepositions were removed. Scores for ranking true match pairs and probabilistic record linkage was performed using Reclink 3.2 software. The descriptive statistics and Kaplan-Meier test were conducted before and after linkage.

Results Of 18511 cancer cases, of both sexes, aged between 0 and 100 years and older, 7328 were true matched pairs. There was an $85.8 \%$ gain in residential address and a $11.0 \%$ gain in death date. In 4086 cases, motheŕs name was aggregated and for all paired registries, cause of death. In the survival analysis, it was verified a $14.4 \%$ underestimation in the one-year survival probability and a $31.6 \%$ underestimation for the three-year.

Conclusion Probabilistic record linkage has shown to be effective in the identification of deaths. An expressive gain in information quality of PBCR-SP database was observed.

\section{P1-494 QUALITY OF PAP SMEAR IN THE PRIMARY CARE UNITS OF SÃO PAULO CITY, BRAZIL}

doi:10.1136/jech.2011.142976g.82

${ }^{1,2} \mathrm{C}$ R P de 0 Longo, ${ }^{1,2} \mathrm{D} \mathrm{M}$ Lopes, ${ }^{1,2} \mathrm{~S}$ V Peres. ${ }^{*}{ }^{1}$ Instituto Israelita de Responsabilidade Social - Hospital Israelita Albert Einstein, Sociedade Beneficente Israelita Albert Einstein, São Paulo, Brazil; ${ }^{2}$ Secretaria Municipal de Saúde, São Paulo, Brazil

Introduction The impact of cervical cancer on morbidity and mortality depends not only on the availability and coverage of screening, but also on the quality of the sample. The aim of this study was to evaluate the quality of Pap smear of the Primary Care Units (PCU), of São Paulo city, Brazil.

Methods This was a cross-sectional study. A sample of 478 Pap smears was selected from 12 PCU-São Paulo-Brazil, between April and September 2010. Collection date, name of the responsible physician, record number, age, pregnancy presence, and previous patient's collections, date of result, presence and epithelial classification of the sample and cytological result were collected from the "registration book". The samples were classified as satisfactory with squamous columnar junction (SCJ), satisfactory without SCJ, and unsatisfactory. Samples without epithelial classification in the "registration book" were reported as "missing". Descriptive analyses were calculated.

Results The mean age was 39 years $(\mathrm{SD}=14.2)$, median 37 years (range 14-82). $38.1 \%$ of samples were satisfactory with SCJ, $22.6 \%$ satisfactory without SCJ, $0.4 \%$ unsatisfactory and $38.9 \%$ missing.

Conclusion It is concluded that there was a low percentage of unsatisfactory samples; however, the results are unreliable, because a high percentage of samples without the epithelial classification was identified. It is necessary to improve the recording of the epithelia by professionals from PCU, because a low quality examination can lead to inadequate prevention of cervical cancer.

\section{P1-495 MEASURING THE PLACEBO EFFECT IN UNBLINDED RANDOMISED COMMUNITY TRIALS}

doi:10.1136/jech.2011.142976g.83

${ }^{1} \mathrm{~N}$ Pierse, ${ }^{*}{ }^{1} \mathrm{M}$ Keall, ${ }^{2} \mathrm{R}$ Arnold, ${ }^{1} \mathrm{P}$ Howden-Chapman. ${ }^{1}$ University of Otago, Wellington, New Zealand; ${ }^{2}$ Victoria University, Wellington, New Zealand

Introduction The double blinded randomised control trial has been developed to provide gold standard estimation of causal effects. However, in many circumstances it is impossible to design studies 